\title{
DESENVOLVIMENTO DE UM MODELO DE PREVISÃO DO TEOR DE ENXOFRE NA DESSULFURAÇÃO DE GUSA EM CARRO TORPEDO DA COMPANHIA SIDERURGICA NACIONAL*
}

\author{
Rafaela Pacheco Malvão dos Santos ${ }^{1}$ \\ Jose Adilson de Castro \\ André Luiz Vasconcellos da Costa e Silva ${ }^{3}$ \\ Antônio Augusto Martins ${ }^{4}$
}

\section{Resumo}

O gusa pode ser dessulfurado em carros torpedos ou em panelas de transferência, já que a dessulfuração do gusa é termodinamicamente favorável, devido as condições redutoras e interação do enxofre e carbono. Dessa maneira, neste trabalho, buscase avaliar a literatura disponível para fornecer subsídios a elaboração de um modelo matemático para a cinética do processo de dessulfuração usado em escala industrial. Foram realizados testes industriais e coletadas amostras de escória e de gusa em quatro momentos do processo de dessulfuração. Dentre os modelos cinéticos disponíveis o modelo de Oeters, foi selecionado por sua simplicidade e adequação às condições da CSN. Com os dados obtidos na amostragem, derivou-se um modelo adaptado do modelo de Oeters para a uma equação de previsão do teor de enxofre ao longo da dessulfuração em carro torpedo.

Palavras chaves: Dessulfuração; Enxofre; Gusa; Modelo matemático.

\section{DEVELOPMENT OF A NEW SULPHUR PREDICTION MODEL FOR THE IRON DESULPHURIZATION PROCESS IN THE TORPEDO CAR AT COMPANHIA SIDERÚRGICA NACIONAL}

\section{Abstract}

This way, hot metal can be desulphurized in torpedo ladle or transfer ladles, since it is thermodynamically very advantageous, due to reducing conditions and carbon sulfur interaction. In this way, this work, seeks to evaluate the available literature to provide subsidies to development of a mathematical model for kinetics of the desulfurization process used in industrial scale. Industrial tests were carried out and slag and hot metal samples were collected in four different times in the desulfurization process. Among several available kinetic models, Oeter's model was selected due it's simplicity and adequate at CSN's conditions. With the data obtained from samples, was derived an adapted model from Oeter's one for a prediction equation of sulfur during desulfurization.

Key words: Desulphurization; Sulfur; hot metal; Mathematical model.

1 Engenheira Metalurgista, Engenheira de Desenvolvimento Sr.,Companhia Siderúrgica Nacional (CSN), Volta Redonda, R.J., Brasil.

2 Engenheiro Metalurgista, Professor Ph.D - Universidade Federal Fluminense (UFF), Volta Redonda, R.J., Brasil.

3 Engenheiro Metalurgista, Professor Ph.D - Universidade Federal Fluminense (UFF), Volta Redonda, R.J., Brasil.

4 Engenheiro Metalurgista, Coordenador de Projetos Especiais, Companhia Siderúrgica Nacional (CSN), Volta Redonda, R.J., Brasil. 


\section{INTRODUÇÃO}

Nos últimos anos, observa-se uma crescente demanda de aços com maior tenacidade e ductilidade. Baixos teores de enxofre são importantes para se atingir tais propriedades. Além disto, o enxofre ou os sulfetos podem ter papel importante em alguns processos de corrosão do aço [1]. Assim, na produção de aços para indústrias de tubos e automobilística, por exemplo, o controle do teor de enxofre é primordial. Para tanto é necessário uma elevada eficiência no processo de dessulfuração, com o menor custo possível. A dessulfuração do gusa é, normalmente, uma alternativa barata e eficiente em relação a dessulfuração do aço em forno panela. A dessulfuração do gusa pode ser realizada em panelas de transferência ou no carro torpedo.

Os teores de enxofre final obtidos nesta operação dependem dos agentes dessulfurantes utilizados, das condições termodinâmicas existentes e, principalmente dos aspectos cinéticos do processo. No caso da dessulfuração do gusa a alta temperatura, as principais etapas controladoras do processo estão ligadas aos mecanismos de transferência de massa, já que as reações químicas são bastante rápidas.

Este trabalho visa a contribuir no desenvolvimento de um modelo de predição do teor de enxofre durante o processo de dessulfuração em carro torpedo permitindo entregar a aciaria um gusa de alta qualidade no menor tempo possível, de forma sistemática e controlada. Para tal, é necessário conhecer o comportamento dos agentes dessulfurantes e a influência dos parâmetros de processo sobre a termodinâmica e a cinética do processo de dessulfuração.

\subsection{Revisão Bibliográfica}

\subsubsection{Desenvolvimento do modelo}

Vários autores modelaram a cinética de injeção de $\mathrm{CaO}$ e $\mathrm{CaC}_{2}$ para a dessulfuração de gusa [2-7].

De acordo com Deo [8] em um sistema metal-escória-gás onde existem várias fases e interfaces e podem ocorrer reações químicas, os processos podem ser controlados pela transferência de massa em cada uma das fases, pelas reações químicas, por dessorção, por absorção, ou pela combinação destes mecanismos.

Oeters [9] formulou um modelo cinético simples para o processo em que partículas permanecem dispersas e em contato com o metal por um determinado tempo. Segundo Oeters, este processo pode ser dividido em duas partes:

1. A microcinética, que descreve a interação de uma partícula com o metal;

2. A macrocinética que descreve a soma das contribuições das partículas e seu efeito sobre o metal líquido.

Oeters assumiu que o transporte de enxofre, $\mathrm{S}$, no metal controla a absorção de enxofre, S, pela partícula.

Logo, na interface partícula-metal o fluxo de enxofre pode ser descrito pela Equação 1:

$j_{s}=\beta_{S}\left[\% S(t)-\% S_{e q}\right]$

Onde: $j_{s}=$ Fluxo de enxofre (número de mols ou átomo-grama, por unidade de tempo e unidade de área);

$\beta_{S}=$ coeficiente de transferência de massa $(\mathrm{m} / \mathrm{s})$;

$\% S(t)=$ teor de enxofre no instante $t(\%)$;

$\% S_{e q}=$ teor de enxofre em equilíbrio (\%). 
É razoável supor que \% $S_{e q}$ seja aproximadamente igual a zero no caso da injeção de $\mathrm{CaO}$ ou $\mathrm{CaC}_{2}$, em função dos equilíbrios (Equações 2 e 3 ):

$\mathrm{CaO}+\mathrm{S}=\mathrm{CaS}+\mathrm{O}$

$\mathrm{CaC}_{2}+\mathrm{S}=\mathrm{CaS}+2 \mathrm{C}$

Logo o fluxo de enxofre $\left(j_{s}\right)$ pode ser aproximado pela equação 4 :

$j_{S}=\beta_{S}[\% S(t)]$

Onde o coeficiente $\beta_{S}$ é ajustado para que o fluxo $\left(j_{s}\right)$ tenha as dimensões gramas $/ \mathrm{m}^{2} . \mathrm{s}$.

Fazendo um balanço de massa do enxofre na partícula, isto é:

[S na partícula em $\mathrm{t}+\mathrm{S}$ que entra na partícula em $\mathrm{dt}=\mathrm{S}$ na partícula em $\mathrm{t}+\mathrm{dt}$ ]

É possível obter-se a Equação 5:

$V \rho[S](t)+j A d t=V \rho[S](t+d t)$

Onde: $V_{p}=$ volume da partícula $\left(\mathrm{m}^{3}\right)$;

$A$ = área da interface $\left(\mathrm{m}^{2}\right)$;

[S] = é a concentração de enxofre em g de enxofre / g de partícula.

E, portanto a Equação 6:

$j \frac{A}{V \rho}=\frac{d[S](t)}{d t}$

Para uma partícula aproximada como uma esfera (Equação 7):

$\frac{A}{V \rho}=\frac{4 \pi r^{2}}{\frac{4}{3} \pi r^{3}}=\frac{3}{r}=\frac{6}{d \rho}$

Onde: $d \rho=$ diâmetro da partícula $(\mathrm{mm})$;

$r=$ raio da partícula $(\mathrm{mm})$.

Substituindo a Equação 7 na Equação 6, temos a Equação 8:

$\beta_{S}[\% S(t)] \frac{6}{d \rho}=\frac{d[S](t)}{d t}$

Se o enxofre do banho é constante durante o tempo de residência de uma partícula no banho, pode-se integrar a Equação 8 obtendo-se a Equação 9:

$\int_{0}^{t_{R}} \beta_{S}[\% S(t)] \frac{6}{d \rho} d t=\int_{o}^{S_{f}} d[S](t)$

Onde: $t_{R}=$ tempo de residência das partículas no metal (s);

$S_{f}=$ teor de enxofre final no gusa (\%).

A concentração de $S$ final de cada partícula pode ser obtida, portanto, pela Equação 10:

$\beta_{S}[\% S(t)] \frac{6}{d \rho} t_{R}=\overline{[S]_{P}}$

Onde: $\overline{[S]_{P}}=$ concentração de enxofre em cada partícula. 
Esta concentração, multiplicada pelo peso de cada partícula, é o peso de S removido por cada partícula. Ou, multiplicando-se esta concentração $\overline{[S]_{P}}$ pela taxa de injeção $\dot{M}_{P}\left(\frac{g}{s}\right)$, diretamente, pode-se obter a taxa de remoção $\overline{[S]_{P}} \dot{M}_{P}$.

$A$ análise macrocinética envolve realizar o balanço de massa do enxofre no gusa (Equações 11, 12 e 13), que permite obter a (Equação 14):

[Sno gusa em $t-$ enxofre removido em $d t=S$ no gusa em $t+d t$ ]

$\frac{M_{g}}{100} \% S(t)-\overline{[S]_{P}} d t=\frac{M_{g}}{100} \% S(t+d t)$

$-\overline{[S]_{P}} d t=\frac{M_{g}}{100}(\% S(t+d t)-\% S(t))$

$-\overline{[S]_{P}} d t=\frac{M_{g}}{100}(d \% S(t))$

$\frac{d \% S(t)}{d t}=-\frac{100}{M_{g}} \overline{[S]_{P}}$

Onde: $M_{g}=$ massa de gusa $(\mathrm{t})$.

Substituindo $\overline{[S}_{P}$ (Equação 10), obtido na análise microcinética, na Equação 14, obtém-se a Equação 15 e 16:

$-\frac{d \% S(t)}{d t}=\frac{100}{M_{g}} \beta_{S}[\% S(t)] \frac{6}{d \rho} t_{R} \dot{M}_{P}$

$-\frac{d \% S(t)}{\% S(t)}=\frac{100}{M_{g}} \beta_{S} \frac{6}{d \rho} t_{R} \dot{M}_{P} d t$

Assumindo a Equação 16 para a constante cinética $k$, tem-se a Equação. 17 e 18:

$k=\frac{100}{M_{g}} \beta_{S} \frac{6}{d \rho} t_{R} \dot{M}_{P}$

$\frac{\% S(t)}{\% S(0)}=\exp (-k t)$

Onde: $\% S(t)=$ teor de enxofre no instante $t(\%)$;

$\% S(0)=$ teor de enxofre no instante $t=0(\%)$;

$k=$ constante cinética de dessulfuração;

$M_{g}=$ massa de gusa ( $\mathrm{t}$ );

$\beta_{S}=$ coeficiente de transferência de massa $(\mathrm{m} / \mathrm{s})$;

$d \rho=$ diâmetro da partícula $(\mathrm{mm})$;

$t_{R}=$ tempo de residência das partículas no banho (s);

$\dot{M}_{P}=$ taxa de injeção $(\mathrm{kg} / \mathrm{min})$;

$t=$ tempo da dessulfuração (s).

A equação 17 descreve a constante cinética de dessulfuração, a qual é diretamente proporcional ao coeficiente de transferência de massa, taxa de injeção e tempo de residência das partículas dessulfurantes no banho e inversamente proporcional a massa de gusa e diâmetro da partícula.

$\mathrm{Na}$ cinética da reação da partícula com o banho, usando a atividade henriana do $\mathrm{S}$ ao invés da \%S temos que o fluxo (Equação 19):

$\mathrm{j}_{\mathrm{S}}=\beta_{\mathrm{S}}[\mathrm{hS}(\mathrm{t})]$ 
Substituindo a atividade henriana pelo coeficiente da atividade henriana, temos a Equação 20:

$\mathrm{j}_{\mathrm{s}}=\beta_{\mathrm{S}}\left[\mathrm{f}_{\mathrm{s}} \% \mathrm{~S}(\mathrm{t})\right]$

O coeficiente da atividade henriana é igual as Equações 21 e 22:

$\operatorname{logf}_{\mathrm{s}}=\mathrm{e}_{\mathrm{S}}^{\mathrm{Si}} \% \mathrm{Si}$

$\mathrm{f}_{\mathrm{s}}=10^{\mathrm{e}_{\mathrm{S}}^{\mathrm{Si}} \% \mathrm{Si}}$

$E$, portanto, o $\beta_{S}$ deve ser corrigido conforme Equação 23:

$$
\beta_{\mathrm{S}} \mathrm{f}_{\mathrm{S}}=\beta_{\mathrm{S}} 10^{\mathrm{e}_{\mathrm{S}}^{\mathrm{Si}} \% \mathrm{Si}}
$$

Onde: $\mathrm{j}_{\mathrm{s}}=$ fluxo de enxofre (número de mols ou átomo-grama, por unidade de tempo e unidade de área);

$\beta_{\mathrm{S}}=$ coeficiente de transferência de massa $(\mathrm{m} / \mathrm{s})$;

$\mathrm{hS}=$ atividade henriana do S;

$\mathrm{f}_{\mathrm{s}}=$ coeficiente da atividade henriana;

$\mathrm{e}_{\mathrm{S}}^{\mathrm{Si}} \% \mathrm{Si}=$ coeficiente de interação do silício com o enxofre.

Havíamos deduzido a Equação 18, na qual a sua constante cinética k é explicitado pela Equação 17. Sendo assim, substituindo $\beta_{S}$ corrigido da Equação 23 na Equação 17, temos a Equação 24:

$\mathrm{k}=\frac{100}{\mathrm{M}_{\mathrm{g}}} \beta_{\mathrm{S}} \mathrm{f}_{\mathrm{S}} \frac{6}{\mathrm{~d} \rho} \mathrm{t}_{\mathrm{R}} \dot{\mathrm{M}}_{\mathrm{P}}=\frac{100}{\mathrm{M}_{\mathrm{g}}} \beta_{\mathrm{S}} 10^{\mathrm{e}_{\mathrm{S}}^{\mathrm{Si}} \% \mathrm{Si}} \frac{6}{\mathrm{~d} \rho} \mathrm{t}_{\mathrm{R}} \dot{\mathrm{M}}_{\mathrm{P}}$

Assim, a dependência de k com o \%Si deve ser da forma (Equação 25):

$\mathrm{k}=\mathrm{k}_{0} 10^{\mathrm{e}_{\mathrm{S}}^{\mathrm{Si}} \% \mathrm{Si}}$

Se assumirmos um comportamento de Arrhenius para o k, teremos (Equação 26):

$\mathrm{k}=\mathrm{k}_{0} \mathrm{e}^{\left(-\frac{\mathrm{E}}{\mathrm{RT}}\right)} 10^{\mathrm{e}_{\mathrm{S}}^{\mathrm{Si}} \% \mathrm{Si}}$

Onde: $\mathrm{k}_{0}=$ constante que engloba $\mathrm{k}$;

$$
\begin{aligned}
& E=\text { energia de ativação }(\mathrm{J} / \mathrm{mol}) ; \\
& R=\text { constante dos gases }\left(8,314 \mathrm{~J} \mathrm{~K}^{-1} \mathrm{~mol}^{-1}\right) \\
& T=\text { temperatura (kelvin) }
\end{aligned}
$$

Simplificadamente, para ajustar o efeito de T (temperatura) e do \%Si (teor de silício) no gusa, teremos que ajustar ko e E. O coeficiente de interação do silício com o enxofre $\left(\mathrm{e}_{\mathrm{S}}^{\mathrm{Si}}\right)$ é de 0.063 [10].

\section{MATERIAIS E MÉTODOS}

Foram feitos experimentos nas estações de dessulfuração de gusa em carro torpedo da Companhia Siderúrgica Nacional.

Em 35 tratamentos (cada tratamento corresponde a um carro torpedo) foram coletadas amostras para análise de enxofre em quatro momentos do processo de dessulfuração, sendo uma amostra inicial, duas intermediárias (com 33\% e 66\% do material injetado) e uma amostra final. Também foram feitas amostragens de escória em 17 corridas sendo uma amostra inicial, uma intermediária (com 50\% do material injetado) e uma amostra final. Através das amostras de escória foram feitos balanços 
de massa do enxofre. O material dessulfurante utilizado em todas as corridas foi uma mistura a base de carbureto de cálcio $\left(\mathrm{CaC}_{2}\right)$.

\section{RESULTADOS E DISCUSSÃO}

Nas amostras de escórias foi analisado o teor de enxofre para verificar a sua partição. $\mathrm{Na}$ Tabela 1, tem-se os valores de enxofre da escória do carro torpedo 24 (corrida 92413 / ciclo 5441). A primeira amostra é a escória antes da dessulfuração, a segunda é a escória coletada aos $50 \%$ e por fim, a terceira é após a dessulfuração.

Tabela 1 - Análise do teor de enxofre na escória nos testes industriais.

\begin{tabular}{|c|c|}
\hline Amostra & $\mathbf{S}$ \\
\hline CT24 (1 $\left.{ }^{a}\right)$ & $0,66 \%$ \\
\hline CT24 (2 $\left.2^{a}\right)$ & $1,97 \%$ \\
\hline CT24 (3) & $3,88 \%$ \\
\hline
\end{tabular}

O aumento do teor de enxofre durante a dessulfuração foi identificada nas amostras de escória como mostrado na Tabela 1. No início da dessulfuração, o \% de enxofre era de 0.66 , aos $50 \%$ o enxofre subiu para $1.97 \%$ e ao final da dessulfuração o valor foi de $3.88 \%$.

Realizou-se um balanço de massa do enxofre para esta escória, como mostrado na Tabela 2.

Tabela 2 - Balanço de massa do enxofre.

\begin{tabular}{|c|c|c|c|c|c|}
\hline Amostra & S escória $(\mathbf{k g})$ & $\%$ g gusa & Gusa (kg) & $\mathrm{S}$ gusa (kg) & Total $(\mathrm{kg})$ \\
\hline CT24 (1 $\left.1^{a}\right)$ & 1,1192 & 0,022 & 268000 & 58,96 & 60,08 \\
\hline CT24 (2a) & 8,0093 & 0,017 & & 45,56 & 53,57 \\
\hline CT24 (3a) & 24,9723 & 0,012 & & 32,16 & 57,13 \\
\hline
\end{tabular}

O balanço de massa não fechou devido à necessidade de se estimar o peso de escória inicial no carro torpedo, pois o Alto-Forno não possui balanças. $\mathrm{O}$ erro foi de 5 a $11 \%$ na amostra em questão.

Mesmo ocorrendo este erro no balanço de massa, ficou evidente a queda de enxofre durante a dessulfuração, não havendo tempo de incubação do enxofre.

Utilizou-se o modelo cinético de Oeters (equação 18) para ajuste de k (coeficiente cinético global da dessulfuração - equação 17).

A equação 18 prevê o teor de enxofre em um determinado momento da dessulfuração, a partir do enxofre inicial $(S(0)$ ), tempo (t) e $k$ (coeficiente cinético global da dessulfuração).

A primeira tentativa foi ajustar a uma única constante cinética $(k)$ a todas as experiências. Observou-se, entretanto, uma dispersão elevada dos resultados.

Analisou-se todos os experimentos de dessulfuração e, pelo seu perfil de queda do teor de enxofre em função do tempo, os dados foram agrupados em 2 famílias, identificados por "círculos" e "quadrados".

Levantou-se o fator k destas famílias "círculos" e "quadrados". Os valores de k obtidos foram de 0,24 e 0,32 respectivamente. Isto indica que há algumas corridas em que a cinética de dessulfuração é mais rápida do que outras. 
Linearizando e separando-se os dados, como mostrado na figura 1, observa-se que as corridas identificadas pelos "quadrados" tem, de fato, cinética de desulfuração mais favorável. Foram estudadas as variáveis destas dessulfurações e a principal diferença observada foi que estas corridas possuem, em média, um teor de silício no gusa de $0,64 \%$ e enquanto as corridas onde a desulfuração foi mais lenta, identificadas pelos "círculos", tem teor médio de silício de $0,46 \%$. Outra diferença fundamental em relação a estas famílias é que a média de temperatura inicial das corridas de cinética mais rápida é $16^{\circ} \mathrm{C}$ superior à média da temperatura das corridas de cinética mais lenta.

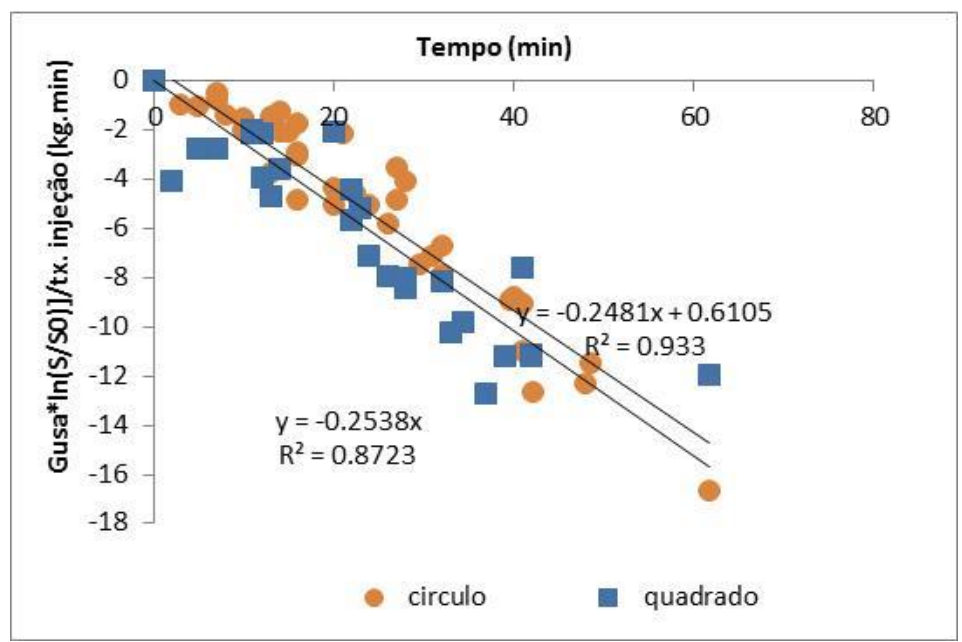

Figura 1 - Valores de kt versus tempo.

Foi verificado a impossibilidade de adotar uma única constante cinética a todas as dessulfurações. Deste modo, foi tomado como base o modelo cinético de Oeters, e feito algumas simplificações e modificações. Foi inserido na sua constante cinética o efeito da temperatura e o teor de silício no gusa, conforme Equação 26.

Através de ferramentas estatísticas, na qual como meta tentou-se atingir o menor erro quadrado da diferença entre os teores de enxofre medidos e estimados, foram ajustados os parâmetros $\mathrm{k} 0$ e $\mathrm{E}$ da constante cinética de dessulfuração na equação 26.

Os parâmetros encontrados foram:

$\mathrm{E}=70479.3$

$\mathrm{k}_{0}=37.1083$

Em vista do conhecimento quantitativo limitado da interação $\mathrm{S} \times \mathrm{Si}$, foi empregado o coeficiente determinado para $1600 \mathrm{C}, \mathrm{e}_{\mathrm{S}}^{\mathrm{Si}}=0.063$ [10].

Por fim, utilizando as constantes $\mathrm{E}, \mathrm{k}_{0} \mathrm{e}_{\mathrm{S}}^{\mathrm{Si}}$ foi obtido o modelo de previsão do teor de enxofre na dessulfuração de gusa em carro torpedo, descrito pela equação 27.

S estimado $=$ Sinicial exp ${ }^{-\left[\left(\frac{\dot{\mathrm{M}}_{\mathrm{P}}}{M g} x k 0 \times \exp ^{\left(-\frac{\mathrm{E}}{\mathrm{RT}}\right)} 10_{\mathrm{e}_{\mathrm{S}} \% \mathrm{Si}}^{\mathrm{Si}_{1}}\right) x t\right]}$

Onde:

ko este parâmetro engloba o coeficiente de transferência de massa, o tempo de residência das partículas dessulfurantes no gusa e o diâmetro inicial da partícula.

$\dot{\mathrm{M}}_{\mathrm{P}}=$ taxa de injeção do agente dessulfurante ( $\left.\mathrm{kg} / \mathrm{min}\right)$;

$M g=$ massa de gusa (t);

$E$ = energia de ativação $(\mathrm{KJ} / \mathrm{mol})$; 
$\mathrm{R}=$ constante dos gases $\left(8,314 \mathrm{~J} \mathrm{~K}^{-1} \mathrm{~mol}^{-1}\right)$;

$\mathrm{T}=$ temperatura inicial (kelvin)

$\mathrm{e}_{\mathrm{S}}^{\mathrm{Si}}=$ coeficiente de interação do silício com o enxofre (0.063);

$\% \mathrm{Si}=\%$ silício no gusa;

$\mathrm{t}=$ tempo de dessulfuração (min).

A figura 2 mostra a comparação dos teores do enxofre medido em vários momentos durante a dessulfuração (tempos $\mathrm{t} 2$, $\mathrm{t} 3$ e t4) versus o enxofre estimado pelo modelo (equação 27).

Onde:

t2 = amostra de gusa retirada aos $33 \%$ do tempo total de dessulfuração;

$\mathrm{t} 3=$ amostra de gusa retirada aos $66 \%$ do tempo total de dessulfuração;

t4 = amostra de gusa retirada ao final da dessulfuração.

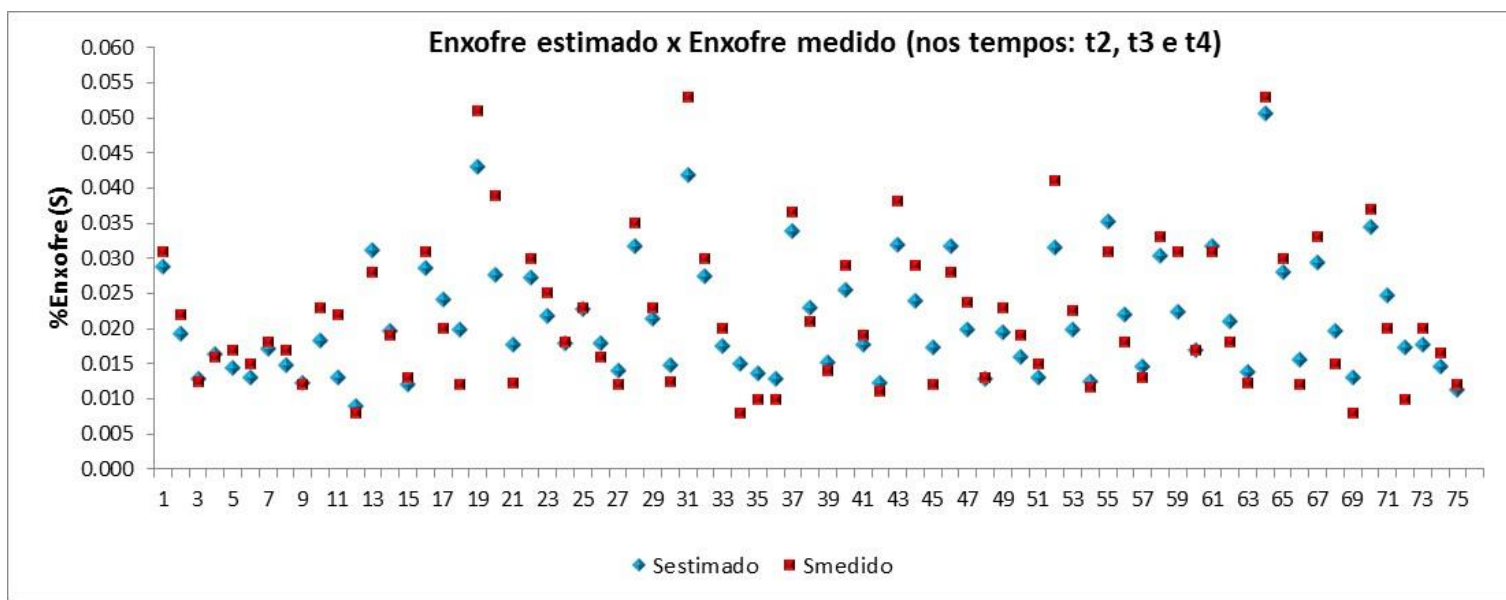

Figura 2 - Valores de enxofre estimado $\mathrm{x}$ enxofre medido nos tempos $\mathrm{t} 2$, t3 e $\mathrm{t} 4$.

Observa-se na figura 2 que o valor estimado é próximo ao valor medido do teor de enxofre.

Para quantificar a qualidade do ajuste do modelo aos dados experimentais, foi construído um histograma (figura 3a) da diferença entre o teor de enxofre estimado e o real (delta S). A média e o desvio padrão das diferenças foram de $-0.000666 \%$ S e $0.00411 \% S$ respectivamente. E realizou-se um teste de hipóteses (figura 3b) para comparar as duas amostras, \%S medido e \%S estimado. O teste identificou pelo intervalo da média que as duas amostras são iguais com $95 \%$ de confiança.
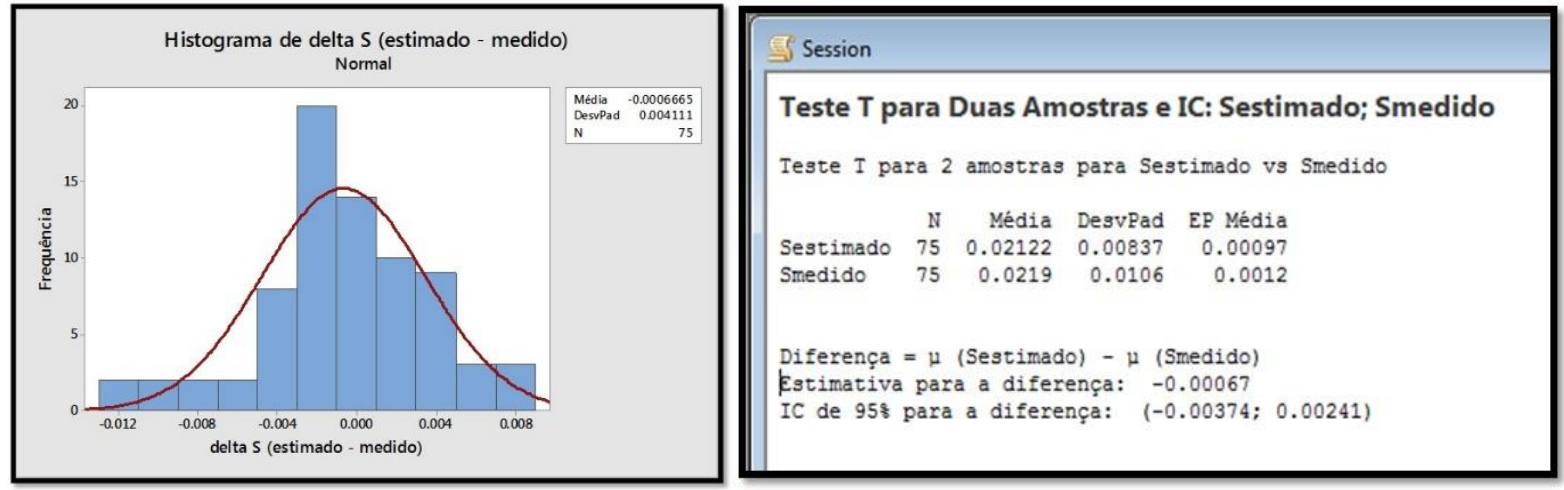

Figura 3(a) - Histograma do delta de enxofre. Figura 3(b) -Teste de hipóteses para duas amostras. 
Como teste e validação do modelo, utilizou-se a equação de previsão do teor de enxofre em um novo banco de dados, que não foi utilizado no processo de ajuste dos coeficientes do modelo. O comportamento dos valores de enxofre medido e estimado comprova o bom ajuste e capacidade preditiva do modelo como mostrado na figura 4 (amostra de enxofre retirada aos 50\% da dessulfuração no tempo t2) e 5 (amostra de enxofre retirada ao final da dessulfuração no tempo t3).

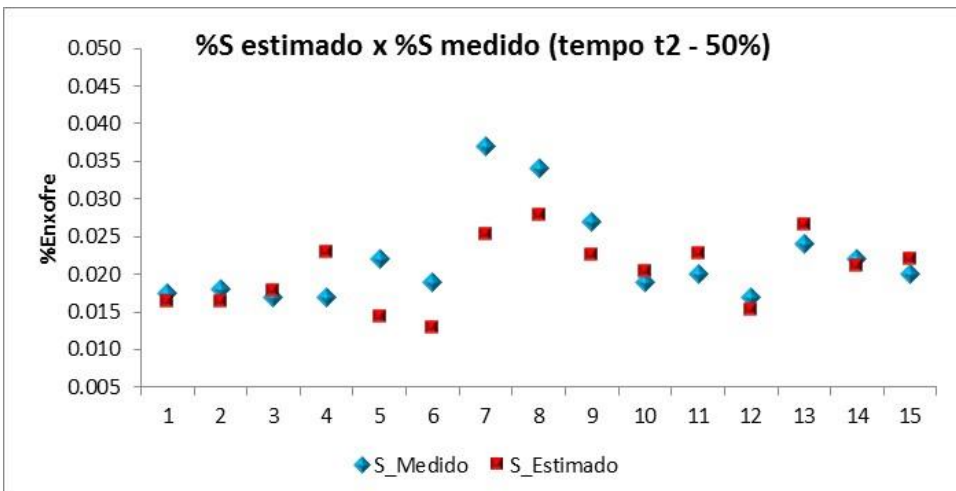

Figura 4 - Valores de enxofre estimado x enxofre medido nos tempos t2.

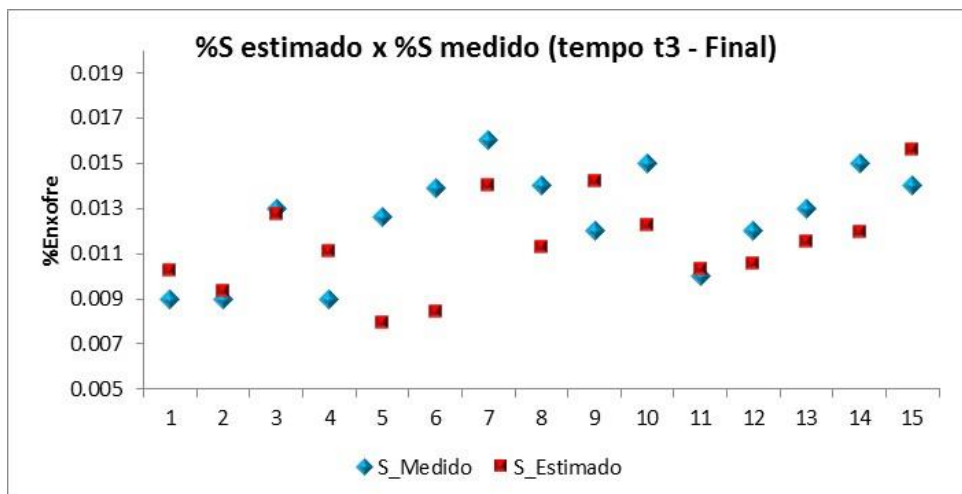

Figura 5 - Valores de enxofre estimado $x$ enxofre medido nos tempos $t 3$.

\section{CONCLUSÃO}

Para descrever a dessulfuração do gusa com injeção de $\mathrm{CaC}_{2}$ no carro torpedo aplicou-se um modelo simples que considera apenas a reação das partículas com 0 gusa, adotando várias simplificações.

Os resultados indicam que, embora a cinética siga, aproximadamente, o tipo de equação proposto, não parece ser possível descrever satisfatoriamente todas as corridas com a mesma constante cinética.

Dois fatores principais foram identificados como afetando a constante cinética, o teor de Si do gusa e a temperatura de tratamento.

As corridas com teor de silício mais alto ou com temperatura mais elevada apresentaram cinética de dessulfuração mais acelerada. Isto é razoável em função da interação termodinâmica Si x S e do evidente efeito da temperatura na cinética de transporte. Para contornar este problema o modelo empírico atual empregado na CSN emprega valores de $\mathrm{k}$ diferentes para diferentes faixas de enxofre no gusa.

Foi proposto um modelo matemático de previsão do teor de enxofre ao longo da dessulfuração, no qual a constante cinética $\mathrm{k}$ leva em consideração a influência do efeito da temperatura e o efeito do teor de silício no gusa. Além disto, são parâmetros do modelo o coeficiente de transferência de massa, o tempo de residência da partícula 
dessulfurante no banho e o diâmetro da partícula, que estão dispostos em uma única variável, assim como a taxa de injeção e o peso de gusa a ser dessulfurado. Ou seja, para cada tratamento de dessulfuração, calcula-se uma constante cinética diferente. Tal modelo obteve uma boa capacidade de previsão. Por este motivo, será implementado no modelo de nível 2, que controla a dessulfuração de gusa em carro torpedo da CSN.

Nas medidas experimentais para o ajuste do modelo, não foi observado um tempo de incubação para a dessulfuração, como relatado por outros autores. Todas as amostras de gusa indicam queda do enxofre com o tempo e aumento da quantidade de enxofre na escória do carro torpedo. Em todas as tentativas de ajuste dos coeficientes de modelamento os resultados indicam a inexistência do tempo de incubação.

\section{REFERÊNCIAS}

1 Pehlke RD, Fuwa T. Control of Sulphur in liquid iron and steel. International Metals Reviews. 1985;30(1):125-40.

2 Varadarajan SESHADRI, C A Silva, I A Silva, P. KROGER. A Kinetic model Applied to the Molten Pig Iron Desulfurization by Injection of Lime-based Powders. ISIJ International. 1997;37(1):21-30.

3 Y. Jin, X.G. Bi, S.R. Yu. KINETIC MODEL FOR POWDER INJECTION DESULF'URIZATION. Acts Metall Sin (Engl Lett). 2006;19(4):258-64.

4 Zhao Y-F, Irons GA. Calcium carbide powder injection into hot metal Part 2 Simultaneous delsulphurisation and deoxidation. Ironmaking and Steelmaking. 1994;21(4):309-17.

5 F.Oeters, P.Strohmenger, W.Pluschkell. Desulfurization kinetics of pig iron with lime and natural gas. Archiv fuer das Eisenhuettenwesen. 1973;44(10):727-33.

6 Brodrick C. Desulphurization of pig iron using calcium carbide based reagents. 7thint Heavy Minerals Conf "What next", Johannesburg, South Africa [Internet]. 2009 [cited 2015 Apr 1]. p. 141-6. Available from: https://saimm.co.za/Conferences/HMC2009/141146 Broderick.pdf

7 Wei Wu, Zhijun Han, Yanbin Hu, Wei Wu. Desulfurizer desulphurization kinetics by the injection method. Journal of University of Science and Technology Beijing. 2008;15(4):375.

8 DEO, B.; BOOM, R. Fundamentals of steelmaking metallurgy. Prentice Hall, 1993.

9 OETERS, F. Kinetic treatment of chemical reactions in emulsion metallurgy. Steel research.Vol.56, n², 1985.

10 Sigworth, GK.; Elliott JF. The thermodynamics of liquid dilute iron alloys. Metal Science. 1974;8:298-310. 